(Supporting Information)

\title{
Michael Addition of Stannyl Ketone Enolate to $\alpha$, $\beta$-Unsaturated Esters Catalyzed by Tetrabutylammonium Bromide and an ab Initio Theoretical Study on the Reaction Course
}

Makoto Yasuda, Kouji Chiba, Noriyuki Ohigashi, Yasuhiro Katoh, and Akio Baba*

Department of Molecular Chemistry and Handai Frontier Research Center, Graduate School of Engineering, Osaka University, 2-1 Yamadaoka, Suita, Osaka 565-0871, Japan and Science and Technology System Division, Ryoka Systems Inc., 1-5-2 Irifune, Urayasu, Chiba 279-0012, Japan

Total energies for all of the calculated species (in hartree). All energies includes zero point vibration energy correction.

$\begin{array}{lr}\text { ester enolate (Scheme 1) } & -267.607889 \\ \text { ketone enolate (Scheme 1) } & -192.418136 \\ \text { enone (Scheme 1) } & -231.063825 \\ \text { enoate (Scheme 1) = 2a } & -306.263640 \\ \text { product in Type A (Scheme 1) } & -498.725096 \\ \text { product in Type B (Scheme 1) } & -423.529637 \\ \text { product in Type C (Scheme 1) } & -573.918737 \\ \text { product in Type D (Scheme 1) } & -498.721569 \\ \mathbf{7} & -507.009657 \\ \mathbf{8} & -507.009812 \\ \mathbf{9} & -390.575233 \\ \mathbf{1 0} & -390.583641 \\ \mathbf{7 - B r} & -3078.526315 \\ \text { A } & -813.244464 \\ \text { B } & -813.283561 \\ \text { C } & -3384.791878 \\ \text { D } & -3384.772850 \\ \text { E } & -3384.778257 \\ \text { F } & -3384.773400 \\ \text { G } & -3384.801164\end{array}$


Geometries (PDB)

\begin{tabular}{|c|c|c|c|c|c|c|c|c|c|}
\hline ester & enol & ate & (Scheme & 1) & & & & & \\
\hline HETATM & 1 & $\mathrm{O} 1$ & UNK & 0 & -1.800 & -0.380 & 1.180 & 1.00 & 0.00 \\
\hline HETATM & 2 & $\mathrm{C} 2$ & UNK & 0 & -0.664 & -0.316 & 0.668 & 1.00 & 0.00 \\
\hline HETATM & 3 & 03 & UNK & 0 & -0.272 & 1.036 & 0.388 & 1.00 & 0.00 \\
\hline HETATM & 4 & C4 & UNK & 0 & 0.976 & 1.272 & -0.196 & 1.00 & 0.00 \\
\hline HETATM & 5 & $\mathrm{C} 5$ & UNK & 0 & 0.224 & -1.336 & 0.352 & 1.00 & 0.00 \\
\hline HETATM & 6 & $7 \mathrm{H}$ & UNK & 0 & 1.068 & 2.360 & -0.324 & 1.00 & 0.00 \\
\hline HETATM & 7 & $8 \mathrm{H}$ & UNK & 0 & 1.076 & 0.788 & -1.180 & 1.00 & 0.00 \\
\hline HETATM & 8 & $9 \mathrm{H}$ & UNK & 0 & 1.804 & 0.920 & 0.440 & 1.00 & 0.00 \\
\hline HETATM & 9 & $\mathrm{OH} 1$ & UNK & 0 & -0.076 & -2.356 & 0.564 & 1.00 & 0.00 \\
\hline HETATM & 10 & $1 \mathrm{H} 1$ & UNK & 0 & 1.200 & -1.180 & -0.096 & 1.00 & 0.00 \\
\hline TER & 11 & & UNK & 0 & & & & & \\
\hline
\end{tabular}

\begin{tabular}{|c|c|c|c|c|c|c|c|c|c|}
\hline ketone & eno & late & ( Sc & & 1) & & & & \\
\hline HETATM & 1 & $\mathrm{C} 1$ & UNK & 0 & -0.324 & 1.460 & -0.072 & 1.00 & 0.00 \\
\hline HETATM & 2 & $\mathrm{C} 2$ & UNK & 0 & -0.468 & -0.068 & -0.000 & 1.00 & 0.00 \\
\hline HETATM & 3 & $\mathrm{C} 3$ & UNK & 0 & 0.684 & -0.820 & -0.164 & 1.00 & 0.00 \\
\hline HETATM & 4 & O4 & UNK & 0 & -1.652 & -0.496 & 0.212 & 1.00 & 0.00 \\
\hline HETATM & 5 & $5 \mathrm{H}$ & UNK & 0 & -0.680 & 1.908 & 0.868 & 1.00 & 0.00 \\
\hline HETATM & 6 & $6 \mathrm{H}$ & UNK & 0 & 0.704 & 1.796 & -0.252 & 1.00 & 0.00 \\
\hline HETATM & 7 & $7 \mathrm{H}$ & UNK & 0 & -0.968 & 1.852 & -0.868 & 1.00 & 0.00 \\
\hline HETATM & 8 & $8 \mathrm{H}$ & UNK & 0 & 1.652 & -0.348 & -0.340 & 1.00 & 0.00 \\
\hline НЕTATM & 9 & $9 \mathrm{H}$ & UNK & 0 & 0.644 & -1.908 & -0.124 & 1.00 & 0.00 \\
\hline TER & 10 & & UNK & 0 & & & & & \\
\hline
\end{tabular}

enone (Scheme 1)

\begin{tabular}{|c|c|c|c|c|c|c|c|c|c|}
\hline HETATM & 1 & $\mathrm{C} 1$ & UNK & 0 & -0.348 & 1.876 & -0.052 & 1.00 & 0.00 \\
\hline HETATM & 2 & $\mathrm{C} 2$ & UNK & 0 & -0.536 & 0.380 & -0.004 & 1.00 & 0.00 \\
\hline HETATM & 3 & C3 & UNK & 0 & 0.696 & -0.436 & 0.180 & 1.00 & 0.00 \\
\hline HETATM & 4 & O4 & UNK & 0 & -1.640 & -0.132 & -0.112 & 1.00 & 0.00 \\
\hline HETATM & 5 & $5 \mathrm{H}$ & UNK & 0 & 0.120 & 2.232 & 0.872 & 1.00 & 0.00 \\
\hline HETATM & 6 & $6 \mathrm{H}$ & UNK & 0 & 0.328 & 2.148 & -0.872 & 1.00 & 0.00 \\
\hline HETATM & 7 & $7 \mathrm{H}$ & UNK & 0 & -1.308 & 2.372 & -0.192 & 1.00 & 0.00 \\
\hline HETATM & 8 & $8 \mathrm{H}$ & UNK & 0 & 1.640 & 0.096 & 0.268 & 1.00 & 0.00 \\
\hline НЕTATM & 9 & $\mathrm{C} 12$ & UNK & 0 & 0.656 & -1.772 & 0.236 & 1.00 & 0.00 \\
\hline HETATM & 10 & $3 \mathrm{HI}$ & UNK & 0 & -0.292 & -2.296 & 0.148 & 1.00 & 0.00 \\
\hline HETATM & 11 & $4 \mathrm{H} 1$ & UNK & 0 & 1.552 & -2.368 & 0.372 & 1.00 & 0.00 \\
\hline TER & 12 & & UNK & 0 & & & & & \\
\hline
\end{tabular}

enoate (Scheme 1) = $2 \mathrm{a}$

$\begin{array}{llllllllll}\text { HETATM } & 1 & \text { C1 } & \text { UNK } & 0 & -0.836 & 1.036 & -1.008 & 1.00 & 0.00\end{array}$ 


\begin{tabular}{|c|c|c|c|c|c|c|c|c|c|}
\hline HETATM & 2 & $\mathrm{C} 2$ & UNK & 0 & -0.800 & 2.372 & -0.992 & 1.00 & 0.00 \\
\hline HETATM & 3 & C3 & UNK & 0 & -0.164 & 0.240 & 0.044 & 1.00 & 0.00 \\
\hline HETATM & 4 & 04 & UNK & 0 & 0.456 & 0.688 & 0.984 & 1.00 & 0.00 \\
\hline HETATM & 5 & 05 & UNK & 0 & -0.336 & -1.080 & -0.176 & 1.00 & 0.00 \\
\hline HETATM & 6 & C6 & UNK & 0 & 0.272 & -1.948 & 0.780 & 1.00 & 0.00 \\
\hline HETATM & 7 & $7 \mathrm{H}$ & UNK & 0 & -1.352 & 0.484 & -1.784 & 1.00 & 0.00 \\
\hline HETATM & 8 & $8 \mathrm{H}$ & UNK & 0 & -0.272 & 2.900 & -0.200 & 1.00 & 0.00 \\
\hline HETATM & 9 & $9 \mathrm{H}$ & UNK & 0 & -1.288 & 2.964 & -1.756 & 1.00 & 0.00 \\
\hline HETATM & 10 & $\mathrm{OH} 1$ & UNK & 0 & -0.132 & -1.764 & 1.780 & 1.00 & 0.00 \\
\hline HETATM & 11 & $1 \mathrm{H} 1$ & UNK & 0 & 0.032 & -2.960 & 0.452 & 1.00 & 0.00 \\
\hline HETATM & 12 & $2 \mathrm{H} 1$ & UNK & 0 & 1.356 & -1.796 & 0.800 & 1.00 & 0.00 \\
\hline TER & 13 & & UNK & 0 & & & & & \\
\hline
\end{tabular}

END

\begin{tabular}{|c|c|c|c|c|c|c|c|c|c|}
\hline Product & in & type & A & (Scheme & 1) & & & & \\
\hline НЕТАТМ & 1 & $\mathrm{O} 1$ & UNK & 0 & -1.384 & 3.548 & 1.676 & 1.00 & 0.00 \\
\hline НЕТАТМ & 2 & $\mathrm{C} 2$ & UNK & 0 & -1.024 & 2.744 & 0.836 & 1.00 & 0.00 \\
\hline HETATM & 3 & 03 & UNK & 0 & -1.460 & 2.932 & -0.432 & 1.00 & 0.00 \\
\hline HETATM & 4 & $\mathrm{C} 4$ & UNK & 0 & -0.976 & 2.116 & -1.512 & 1.00 & 0.00 \\
\hline HETATM & 5 & $\mathrm{C} 5$ & UNK & 0 & -0.212 & 1.516 & 1.144 & 1.00 & 0.00 \\
\hline HETATM & 6 & $7 \mathrm{H}$ & UNK & 0 & -1.696 & 2.256 & -2.320 & 1.00 & 0.00 \\
\hline HETATM & 7 & $8 \mathrm{H}$ & UNK & 0 & -0.892 & 1.048 & -1.264 & 1.00 & 0.00 \\
\hline HETATM & 8 & $9 \mathrm{H}$ & UNK & 0 & 0.000 & 2.492 & -1.840 & 1.00 & 0.00 \\
\hline HETATM & 9 & $\mathrm{OH} 1$ & UNK & 0 & 0.484 & 1.276 & 0.336 & 1.00 & 0.00 \\
\hline HETATM & 10 & $1 \mathrm{H} 1$ & UNK & 0 & 0.360 & 1.720 & 2.052 & 1.00 & 0.00 \\
\hline HETATM & 11 & C12 & UNK & 0 & -1.112 & 0.256 & 1.364 & 1.00 & 0.00 \\
\hline HETATM & 12 & $\mathrm{C} 13$ & UNK & 0 & -0.344 & -1.024 & 1.324 & 1.00 & 0.00 \\
\hline HETATM & 13 & $\mathrm{C} 14$ & UNK & 0 & 0.104 & -1.508 & 0.108 & 1.00 & 0.00 \\
\hline HETATM & 14 & $\mathrm{C} 15$ & UNK & 0 & 0.892 & -2.820 & 0.080 & 1.00 & 0.00 \\
\hline HETATM & 15 & 016 & UNK & 0 & -0.092 & -0.940 & -1.024 & 1.00 & 0.00 \\
\hline HETATM & 16 & $7 \mathrm{H1}$ & UNK & 0 & -1.640 & 0.388 & 2.320 & 1.00 & 0.00 \\
\hline НETATM & 17 & $8 \mathrm{H1}$ & UNK & 0 & -1.880 & 0.252 & 0.576 & 1.00 & 0.00 \\
\hline НETATM & 18 & $9 \mathrm{H} 1$ & UNK & 0 & -0.112 & -1.544 & 2.252 & 1.00 & 0.00 \\
\hline НETATM & 19 & OH2 & UNK & 0 & 0.376 & -3.548 & -0.556 & 1.00 & 0.00 \\
\hline НETATM & 20 & $1 \mathrm{H} 2$ & UNK & 0 & 1.884 & -2.648 & -0.364 & 1.00 & 0.00 \\
\hline HETATM & 21 & $2 \mathrm{H} 2$ & UNK & 0 & 1.028 & -3.260 & 1.080 & 1.00 & 0.00 \\
\hline TER & 22 & & UNK & 0 & & & & & \\
\hline
\end{tabular}

END

$\begin{array}{lccccrrrrr}\text { Product } & \text { in } & \text { type } & \text { B } & \text { (Scheme } & \text { 1) } & & & & \\ \text { HETATM } & 1 & \text { C1 } & \text { UNK } & 0 & -2.836 & 0.828 & -0.428 & 1.00 & 0.00 \\ \text { HETATM } & 2 & \text { C2 } & \text { UNK } & 0 & -1.736 & 1.856 & -0.216 & 1.00 & 0.00 \\ \text { HETATM } & 3 & \text { C3 } & \text { UNK } & 0 & -0.416 & 1.320 & 0.244 & 1.00 & 0.00 \\ \text { HETATM } & 4 & \text { C4 } & \text { UNK } & 0 & 0.420 & 0.728 & -0.944 & 1.00 & 0.00 \\ \text { HETATM } & 5 & \text { C5 } & \text { UNK } & 0 & 1.608 & -0.052 & -0.480 & 1.00 & 0.00 \\ \text { HETATM } & 6 & \text { C6 } & \text { UNK } & 0 & 1.432 & -1.308 & 0.068 & 1.00 & 0.00\end{array}$




$\begin{array}{lrrlrrrrrr}\text { HETATM } & 7 & \text { C7 } & \text { UNK } & 0 & 2.668 & -2.080 & 0.536 & 1.00 & 0.00 \\ \text { HETATM } & 8 & \text { O8 } & \text { UNK } & 0 & -1.940 & 3.044 & -0.460 & 1.00 & 0.00 \\ \text { HETATM } & 9 & \text { O9 } & \text { UNK } & 0 & 0.304 & -1.896 & 0.212 & 1.00 & 0.00 \\ \text { HETATM } & 10 & 0 \mathrm{H1} & \text { UNK } & 0 & -2.432 & -0.116 & -0.800 & 1.00 & 0.00 \\ \text { HETATM } & 11 & 1 \mathrm{H1} & \text { UNK } & 0 & -3.296 & 0.608 & 0.544 & 1.00 & 0.00 \\ \text { HETATM } & 12 & 2 \mathrm{H1} & \text { UNK } & 0 & -3.608 & 1.220 & -1.096 & 1.00 & 0.00 \\ \text { HETATM } & 13 & 3 \mathrm{H1} & \text { UNK } & 0 & 0.152 & 2.116 & 0.728 & 1.00 & 0.00 \\ \text { HETATM } & 14 & 4 \mathrm{H} 1 & \text { UNK } & 0 & -0.568 & 0.480 & 0.936 & 1.00 & 0.00 \\ \text { HETATM } & 15 & 5 \mathrm{H} 1 & \text { UNK } & 0 & -0.244 & 0.068 & -1.520 & 1.00 & 0.00 \\ \text { HETATM } & 16 & 6 \mathrm{H1} & \text { UNK } & 0 & 0.708 & 1.560 & -1.604 & 1.00 & 0.00 \\ \text { HETATM } & 17 & 7 \mathrm{H} 1 & \text { UNK } & 0 & 2.596 & 0.408 & -0.524 & 1.00 & 0.00 \\ \text { HETATM } & 18 & 8 \mathrm{H} 1 & \text { UNK } & 0 & 2.732 & -3.040 & 0.004 & 1.00 & 0.00 \\ \text { HETATM } & 19 & 9 \mathrm{H1} & \text { UNK } & 0 & 3.608 & -1.528 & 0.376 & 1.00 & 0.00 \\ \text { HETATM } & 20 & 0 \mathrm{H} 2 & \text { UNK } & 0 & 2.576 & -2.316 & 1.604 & 1.00 & 0.00 \\ \text { TER } & 21 & & \text { UNK } & 0 & & & & & \\ \text { END } & & & & & & & & \end{array}$

\begin{tabular}{|c|c|c|c|c|c|c|c|c|c|}
\hline Product & in & type & C & (Scheme & 1) & & & & \\
\hline HETATM & 1 & $\mathrm{C} 1$ & UNK & 0 & 0.576 & -1.308 & -1.112 & 1.00 & 0.00 \\
\hline HETATM & 2 & $\mathrm{C} 2$ & UNK & 0 & 1.384 & 0.040 & -0.956 & 1.00 & 0.00 \\
\hline HETATM & 3 & $\mathrm{C} 3$ & UNK & 0 & 1.208 & -2.432 & -0.364 & 1.00 & 0.00 \\
\hline HETATM & 4 & O4 & UNK & 0 & 2.020 & -3.232 & -0.800 & 1.00 & 0.00 \\
\hline HETATM & 5 & 05 & UNK & 0 & 0.844 & -2.444 & 0.952 & 1.00 & 0.00 \\
\hline HETATM & 6 & $\mathrm{C} 6$ & UNK & 0 & 1.504 & -3.412 & 1.760 & 1.00 & 0.00 \\
\hline HETATM & 7 & $7 \mathrm{H}$ & UNK & 0 & 0.548 & -1.572 & -2.168 & 1.00 & 0.00 \\
\hline HETATM & 8 & $8 \mathrm{H}$ & UNK & 0 & 1.360 & 0.296 & 0.116 & 1.00 & 0.00 \\
\hline HETATM & 9 & $9 \mathrm{H}$ & UNK & 0 & 2.428 & -0.172 & -1.220 & 1.00 & 0.00 \\
\hline HETATM & 10 & $\mathrm{OH} 1$ & UNK & 0 & 2.588 & -3.260 & 1.748 & 1.00 & 0.00 \\
\hline HETATM & 11 & $1 \mathrm{H} 1$ & UNK & 0 & 1.112 & -3.268 & 2.768 & 1.00 & 0.00 \\
\hline HETATM & 12 & $2 \mathrm{H} 1$ & UNK & 0 & 1.288 & -4.428 & 1.408 & 1.00 & 0.00 \\
\hline HETATM & 13 & C13 & UNK & 0 & 0.836 & 1.160 & -1.768 & 1.00 & 0.00 \\
\hline HETATM & 14 & C14 & UNK & 0 & -0.216 & 1.924 & -1.300 & 1.00 & 0.00 \\
\hline HETATM & 15 & 015 & UNK & 0 & -0.832 & 1.860 & -0.212 & 1.00 & 0.00 \\
\hline HETATM & 16 & 016 & UNK & 0 & -0.628 & 2.924 & -2.232 & 1.00 & 0.00 \\
\hline HETATM & 17 & $\mathrm{C} 17$ & UNK & 0 & -1.680 & 3.740 & -1.788 & 1.00 & 0.00 \\
\hline HETATM & 18 & $8 \mathrm{H1}$ & UNK & 0 & -0.436 & -1.116 & -0.752 & 1.00 & 0.00 \\
\hline HETATM & 19 & $9 \mathrm{H1}$ & UNK & 0 & 1.212 & 1.324 & -2.772 & 1.00 & 0.00 \\
\hline HETATM & 20 & $1 \mathrm{H} 2$ & UNK & 0 & -2.584 & 3.160 & -1.544 & 1.00 & 0.00 \\
\hline HETATM & 21 & $2 \mathrm{H} 2$ & UNK & 0 & -1.408 & 4.316 & -0.892 & 1.00 & 0.00 \\
\hline HETATM & 22 & $3 \mathrm{H} 2$ & UNK & 0 & -1.896 & 4.432 & -2.608 & 1.00 & 0.00 \\
\hline TER & 23 & & UNK & 0 & & & & & \\
\hline
\end{tabular}

END

Product in type $D$ (Scheme 1)

$\begin{array}{llllllllll}\text { HETATM } & 1 & \text { C1 } & \text { UNK } & 0 & 0.720 & -1.876 & -0.696 & 1.00 & 0.00\end{array}$




\begin{tabular}{|c|c|c|c|c|c|c|c|c|c|}
\hline HETATM & 3 & $\mathrm{C} 3$ & UNK & 0 & 1.236 & -2.964 & 0.192 & 1.00 & 0.00 \\
\hline HETATM & 4 & $\mathrm{O} 4$ & UNK & 0 & 2.048 & -3.808 & -0.176 & 1.00 & 0.00 \\
\hline HETATM & 5 & C5 & UNK & 0 & 0.748 & -2.944 & 1.632 & 1.00 & 0.00 \\
\hline HETATM & 6 & $7 \mathrm{H}$ & UNK & 0 & 0.796 & -2.188 & -1.740 & 1.00 & 0.00 \\
\hline HETATM & 7 & $8 \mathrm{H}$ & UNK & 0 & 1.532 & -0.300 & 0.564 & 1.00 & 0.00 \\
\hline HETATM & 8 & $9 \mathrm{H}$ & UNK & 0 & 2.568 & -0.728 & -0.804 & 1.00 & 0.00 \\
\hline HETATM & 9 & $\mathrm{C} 13$ & UNK & 0 & 0.964 & 0.616 & -1.268 & 1.00 & 0.00 \\
\hline HETATM & 10 & C14 & UNK & 0 & -0.136 & 1.292 & -0.768 & 1.00 & 0.00 \\
\hline HETATM & 11 & 015 & UNK & 0 & -0.760 & 1.100 & 0.304 & 1.00 & 0.00 \\
\hline HETATM & 12 & 016 & UNK & 0 & -0.592 & 2.340 & -1.620 & 1.00 & 0.00 \\
\hline HETATM & 13 & C17 & UNK & 0 & -1.688 & 3.060 & -1.124 & 1.00 & 0.00 \\
\hline HETATM & 14 & $8 \mathrm{H1}$ & UNK & 0 & -0.316 & -1.636 & -0.456 & 1.00 & 0.00 \\
\hline HETATM & 15 & $9 \mathrm{H} 1$ & UNK & 0 & 1.344 & 0.864 & -2.256 & 1.00 & 0.00 \\
\hline HETATM & 16 & $1 \mathrm{H} 2$ & UNK & 0 & -2.564 & 2.420 & -0.956 & 1.00 & 0.00 \\
\hline HETATM & 17 & $2 \mathrm{H} 2$ & UNK & 0 & -1.460 & 3.564 & -0.176 & 1.00 & 0.00 \\
\hline HETATM & 18 & $3 \mathrm{H} 2$ & UNK & 0 & -1.928 & 3.812 & -1.888 & 1.00 & 0.00 \\
\hline HETATM & 19 & $4 \mathrm{H} 2$ & UNK & 0 & 0.760 & -1.920 & 2.032 & 1.00 & 0.00 \\
\hline HETATM & 20 & $5 \mathrm{H} 2$ & UNK & 0 & -0.292 & -3.276 & 1.664 & 1.00 & 0.00 \\
\hline HETATM & 21 & $6 \mathrm{H} 2$ & UNK & 0 & 1.360 & -3.608 & 2.252 & 1.00 & 0.00 \\
\hline $\mathrm{TER}$ & 22 & & UNK & 0 & & & & & \\
\hline \multicolumn{10}{|l|}{ END } \\
\hline \multicolumn{10}{|l|}{7} \\
\hline HETATM & 1 & $\mathrm{C} 1$ & UNK & 0 & 3.324 & -2.068 & -2.260 & 1.00 & 0.00 \\
\hline HETATM & 2 & $2 \mathrm{H}$ & UNK & 0 & 4.372 & -2.020 & -2.552 & 1.00 & 0.00 \\
\hline HETATM & 3 & $\mathrm{C} 3$ & UNK & 0 & 2.812 & -1.112 & -1.384 & 1.00 & 0.00 \\
\hline HETATM & 4 & $4 \mathrm{H}$ & UNK & 0 & 3.464 & -0.324 & -1.020 & 1.00 & 0.00 \\
\hline HETATM & 5 & C5 & UNK & 0 & 1.464 & -1.140 & -0.996 & 1.00 & 0.00 \\
\hline HETATM & 6 & C7 & UNK & 0 & 0.640 & -2.140 & -1.532 & 1.00 & 0.00 \\
\hline HETATM & 7 & $8 \mathrm{H}$ & UNK & 0 & -0.408 & -2.156 & -1.252 & 1.00 & 0.00 \\
\hline HETATM & 8 & C9 & UNK & 0 & 1.152 & -3.096 & -2.408 & 1.00 & 0.00 \\
\hline HETATM & 9 & OH1 & UNK & 0 & 0.492 & -3.868 & -2.804 & 1.00 & 0.00 \\
\hline HETATM & 10 & $\mathrm{C} 11$ & UNK & 0 & 2.496 & -3.068 & -2.772 & 1.00 & 0.00 \\
\hline HETATM & 11 & $2 \mathrm{H1}$ & UNK & 0 & 2.896 & -3.812 & -3.460 & 1.00 & 0.00 \\
\hline HETATM & 12 & $\mathrm{C} 13$ & UNK & 0 & 0.896 & -0.148 & -0.044 & 1.00 & 0.00 \\
\hline HETATM & 13 & $\mathrm{C} 14$ & UNK & 0 & 1.656 & 0.564 & 0.812 & 1.00 & 0.00 \\
\hline HETATM & 14 & 015 & UNK & 0 & -0.444 & -0.068 & -0.120 & 1.00 & 0.00 \\
\hline HETATM & 15 & $6 \mathrm{H1}$ & UNK & 0 & 2.728 & 0.424 & 0.872 & 1.00 & 0.00 \\
\hline HETATM & 16 & $7 \mathrm{H1}$ & UNK & 0 & 1.224 & 1.284 & 1.500 & 1.00 & 0.00 \\
\hline HETATM & 17 & SN18 & UNK & 0 & -1.672 & 1.164 & 0.836 & 1.00 & 0.00 \\
\hline HETATM & 18 & C19 & UNK & 0 & -1.188 & 3.160 & 0.228 & 1.00 & 0.00 \\
\hline HETATM & 19 & $\mathrm{C} 2 \mathrm{O}$ & UNK & 0 & -1.476 & 0.836 & 2.944 & 1.00 & 0.00 \\
\hline HETATM & 20 & $\mathrm{C} 21$ & UNK & 0 & -3.548 & 0.492 & 0.072 & 1.00 & 0.00 \\
\hline HETATM & 21 & $2 \mathrm{H} 2$ & UNK & 0 & -1.912 & 3.872 & 0.644 & 1.00 & 0.00 \\
\hline HETATM & 22 & $3 \mathrm{H} 2$ & UNK & 0 & -0.188 & 3.448 & 0.564 & 1.00 & 0.00 \\
\hline HETATM & 23 & $4 \mathrm{H} 2$ & UNK & 0 & -1.220 & 3.236 & -0.864 & 1.00 & 0.00 \\
\hline
\end{tabular}




$\begin{array}{lrlllrrrrr}\text { HETATM } & 24 & 5 \mathrm{H} 2 & \text { UNK } & 0 & -2.404 & 1.108 & 3.456 & 1.00 & 0.00 \\ \text { HETATM } & 25 & 6 \mathrm{H} 2 & \text { UNK } & 0 & -1.276 & -0.220 & 3.136 & 1.00 & 0.00 \\ \text { HETATM } & 26 & 7 \mathrm{H} 2 & \text { UNK } & 0 & -0.660 & 1.424 & 3.368 & 1.00 & 0.00 \\ \text { HETATM } & 27 & 8 \mathrm{H} 2 & \text { UNK } & 0 & -4.368 & 1.120 & 0.432 & 1.00 & 0.00 \\ \text { HETATM } & 28 & 9 \mathrm{H} 2 & \text { UNK } & 0 & -3.540 & 0.524 & -1.024 & 1.00 & 0.00 \\ \text { HETATM } & 29 & 0 \mathrm{H} 3 & \text { UNK } & 0 & -3.740 & -0.536 & 0.384 & 1.00 & 0.00 \\ \text { TER } & 30 & & \text { UNK } & 0 & & & & & \end{array}$

END

8

\begin{tabular}{|c|c|c|c|c|c|c|c|c|c|}
\hline HETATM & 1 & $\mathrm{C} 1$ & UNK & 0 & 2.288 & -2.624 & -1.048 & 1.00 & 0.00 \\
\hline HETATM & 2 & $2 \mathrm{H}$ & UNK & 0 & 2.684 & -3.376 & -0.368 & 1.00 & 0.00 \\
\hline НЕТАТМ & 3 & C3 & UNK & 0 & 2.012 & -1.340 & -0.580 & 1.00 & 0.00 \\
\hline НЕТАТМ & 4 & $4 \mathrm{H}$ & UNK & 0 & 2.204 & -1.116 & 0.464 & 1.00 & 0.00 \\
\hline НЕTATM & 5 & $\mathrm{C} 5$ & UNK & 0 & 1.496 & -0.360 & -1.440 & 1.00 & 0.00 \\
\hline HETATM & 6 & $\mathrm{C} 7$ & UNK & 0 & 1.264 & -0.696 & -2.784 & 1.00 & 0.00 \\
\hline HETATM & 7 & $8 \mathrm{H}$ & UNK & 0 & 0.864 & 0.072 & -3.440 & 1.00 & 0.00 \\
\hline HETATM & 8 & $\mathrm{C9}$ & UNK & 0 & 1.544 & -1.976 & -3.252 & 1.00 & 0.00 \\
\hline HETATM & 9 & $\mathrm{OH1}$ & UNK & 0 & 1.360 & -2.220 & -4.296 & 1.00 & 0.00 \\
\hline HETATM & 10 & C11 & UNK & 0 & 2.052 & -2.944 & -2.388 & 1.00 & 0.00 \\
\hline HETATM & 11 & $2 \mathrm{H1}$ & UNK & 0 & 2.272 & -3.948 & -2.752 & 1.00 & 0.00 \\
\hline HETATM & 12 & C13 & UNK & 0 & 1.172 & 1.040 & -1.004 & 1.00 & 0.00 \\
\hline HETATM & 13 & $\mathrm{C} 14$ & UNK & 0 & 1.392 & 1.452 & 0.412 & 1.00 & 0.00 \\
\hline HETATM & 14 & 015 & UNK & 0 & 0.696 & 1.828 & -1.820 & 1.00 & 0.00 \\
\hline HETATM & 15 & $6 \mathrm{H1}$ & UNK & 0 & 2.172 & 0.892 & 0.932 & 1.00 & 0.00 \\
\hline HETATM & 16 & $7 \mathrm{H1}$ & UNK & 0 & 1.644 & 2.516 & 0.424 & 1.00 & 0.00 \\
\hline HETATM & 17 & SN18 & UNK & 0 & -0.472 & 1.324 & 1.572 & 1.00 & 0.00 \\
\hline HETATM & 18 & C19 & UNK & 0 & -1.660 & 3.036 & 1.056 & 1.00 & 0.00 \\
\hline HETATM & 19 & $\mathrm{C} 20$ & UNK & 0 & 0.104 & 1.368 & 3.644 & 1.00 & 0.00 \\
\hline НЕТАТМ & 20 & $\mathrm{C} 21$ & UNK & 0 & -1.512 & -0.496 & 1.120 & 1.00 & 0.00 \\
\hline HETATM & 21 & $2 \mathrm{H} 2$ & UNK & 0 & -2.680 & 2.932 & 1.432 & 1.00 & 0.00 \\
\hline HETATM & 22 & $3 \mathrm{H} 2$ & UNK & 0 & -1.224 & 3.952 & 1.468 & 1.00 & 0.00 \\
\hline HETATM & 23 & $4 \mathrm{H} 2$ & UNK & 0 & -1.700 & 3.140 & -0.036 & 1.00 & 0.00 \\
\hline HETATM & 24 & $5 \mathrm{H} 2$ & UNK & 0 & -0.780 & 1.376 & 4.292 & 1.00 & 0.00 \\
\hline HETATM & 25 & $6 \mathrm{H} 2$ & UNK & 0 & 0.704 & 0.488 & 3.904 & 1.00 & 0.00 \\
\hline HETATM & 26 & $7 \mathrm{H} 2$ & UNK & 0 & 0.696 & 2.264 & 3.868 & 1.00 & 0.00 \\
\hline HETATM & 27 & $8 \mathrm{H} 2$ & UNK & 0 & -1.728 & -0.564 & 0.048 & 1.00 & 0.00 \\
\hline НETATM & 28 & $9 \mathrm{H} 2$ & UNK & 0 & -0.920 & -1.376 & 1.404 & 1.00 & 0.00 \\
\hline HETATM & 29 & OH3 & UNK & 0 & -2.460 & -0.532 & 1.664 & 1.00 & 0.00 \\
\hline TER & 30 & & UNK & 0 & & & & & \\
\hline
\end{tabular}

END

9

\begin{tabular}{|c|c|c|c|c|c|c|c|c|c|}
\hline HETATM & 1 & SN18 & UNK & 0 & -1.104 & 0.596 & 0.068 & 1.00 & 0.00 \\
\hline HETATM & 2 & C19 & UNK & 0 & -1.356 & 2.548 & -0.748 & 1.00 & 0.00 \\
\hline НЕTATM & 3 & $\mathrm{C} 20$ & UNK & 0 & -0.544 & 0.620 & 2.136 & 1.00 & 0.00 \\
\hline
\end{tabular}




\begin{tabular}{|c|c|c|c|c|c|c|c|c|c|}
\hline HETATM & 4 & C21 & UNK & 0 & -2.744 & -0.712 & -0.360 & 1.00 & 0.00 \\
\hline HETATM & 5 & $2 \mathrm{H} 2$ & UNK & 0 & -2.200 & 3.072 & -0.284 & 1.00 & 0.00 \\
\hline HETATM & 6 & $3 \mathrm{H} 2$ & UNK & 0 & -0.448 & 3.144 & -0.592 & 1.00 & 0.00 \\
\hline HETATM & 7 & $4 \mathrm{H} 2$ & UNK & 0 & -1.540 & 2.484 & -1.828 & 1.00 & 0.00 \\
\hline HETATM & 8 & $5 \mathrm{H} 2$ & UNK & 0 & -1.308 & 1.136 & 2.732 & 1.00 & 0.00 \\
\hline HETATM & 9 & $6 \mathrm{H} 2$ & UNK & 0 & -0.428 & -0.392 & 2.528 & 1.00 & 0.00 \\
\hline HETATM & 10 & $7 \mathrm{H} 2$ & UNK & 0 & 0.404 & 1.148 & 2.260 & 1.00 & 0.00 \\
\hline HETATM & 11 & $8 \mathrm{H} 2$ & UNK & 0 & -2.852 & -0.828 & -1.444 & 1.00 & 0.00 \\
\hline HETATM & 12 & $9 \mathrm{H} 2$ & UNK & 0 & -2.584 & -1.700 & 0.076 & 1.00 & 0.00 \\
\hline HETATM & 13 & ОН3 & UNK & 0 & -3.676 & -0.304 & 0.040 & 1.00 & 0.00 \\
\hline HETATM & 14 & 031 & UNK & 0 & 0.460 & -0.016 & -0.976 & 1.00 & 0.00 \\
\hline HETATM & 15 & C32 & UNK & 0 & 1.108 & -1.172 & -0.936 & 1.00 & 0.00 \\
\hline HETATM & 16 & C33 & UNK & 0 & 0.832 & -2.228 & -0.140 & 1.00 & 0.00 \\
\hline HETATM & 17 & O34 & UNK & 0 & 2.116 & -1.124 & -1.840 & 1.00 & 0.00 \\
\hline HETATM & 18 & C35 & UNK & 0 & 2.944 & -2.264 & -1.964 & 1.00 & 0.00 \\
\hline HETATM & 19 & $6 \mathrm{H3}$ & UNK & 0 & 0.012 & -2.192 & 0.560 & 1.00 & 0.00 \\
\hline HETATM & 20 & $7 \mathrm{H} 3$ & UNK & 0 & 1.412 & -3.144 & -0.164 & 1.00 & 0.00 \\
\hline HETATM & 21 & $8 \mathrm{H3}$ & UNK & 0 & 3.460 & -2.484 & -1.024 & 1.00 & 0.00 \\
\hline HETATM & 22 & $9 \mathrm{H3}$ & UNK & 0 & 2.364 & -3.140 & -2.276 & 1.00 & 0.00 \\
\hline HETATM & 23 & $\mathrm{OH} 4$ & UNK & 0 & 3.676 & -2.016 & -2.736 & 1.00 & 0.00 \\
\hline TER & 24 & & UNK & 0 & & & & & \\
\hline
\end{tabular}

END

$\begin{array}{lrrll}10 & & & & \\ \text { HETATM } & 1 & \text { SN18 } & \text { UNK } & 0 \\ \text { HETATM } & 2 & \text { C19 } & \text { UNK } & 0 \\ \text { HETATM } & 3 & \text { C20 } & \text { UNK } & 0 \\ \text { HETATM } & 4 & \text { C21 } & \text { UNK } & 0 \\ \text { HETATM } & 5 & 2 \mathrm{H} 2 & \text { UNK } & 0 \\ \text { HETATM } & 6 & 3 \mathrm{H} 2 & \text { UNK } & 0 \\ \text { HETATM } & 7 & 4 \mathrm{H} 2 & \text { UNK } & 0 \\ \text { HETATM } & 8 & 5 \mathrm{H} 2 & \text { UNK } & 0 \\ \text { HETATM } & 9 & 6 \mathrm{H} 2 & \text { UNK } & 0 \\ \text { HETATM } & 10 & 7 \mathrm{H} 2 & \text { UNK } & 0 \\ \text { HETATM } & 11 & 8 \mathrm{H} 2 & \text { UNK } & 0 \\ \text { HETATM } & 12 & 9 \mathrm{H} 2 & \text { UNK } & 0 \\ \text { HETATM } & 13 & 0 \mathrm{H} 3 & \text { UNK } & 0 \\ \text { HETATM } & 14 & \text { O31 } & \text { UNK } & 0 \\ \text { HETATM } & 15 & \text { C32 } & \text { UNK } & 0 \\ \text { HETATM } & 16 & \text { C33 } & \text { UNK } & 0 \\ \text { HETATM } & 17 & \text { O34 } & \text { UNK } & 0 \\ \text { HETATM } & 18 & \text { C35 } & \text { UNK } & 0 \\ \text { HETATM } & 19 & 6 \mathrm{H} 3 & \text { UNK } & 0 \\ \text { HETATM } & 20 & 7 \mathrm{H} 3 & \text { UNK } & 0 \\ \text { HETATM } & 21 & 8 \mathrm{H} 3 & \text { UNK } & 0 \\ \text { HETATM } & 22 & 9 \mathrm{H} 3 & \text { UNK } & 0\end{array}$

$\begin{array}{rrrrr}-1.092 & 0.404 & 0.068 & 1.00 & 0.00 \\ -1.252 & 2.212 & -1.072 & 1.00 & 0.00 \\ -0.624 & 0.768 & 2.132 & 1.00 & 0.00 \\ -2.944 & -0.696 & -0.064 & 1.00 & 0.00 \\ -2.028 & 2.864 & -0.660 & 1.00 & 0.00 \\ -0.296 & 2.744 & -1.052 & 1.00 & 0.00 \\ -1.508 & 1.992 & -2.116 & 1.00 & 0.00 \\ -1.388 & 1.400 & 2.596 & 1.00 & 0.00 \\ -0.568 & -0.168 & 2.692 & 1.00 & 0.00 \\ 0.340 & 1.280 & 2.204 & 1.00 & 0.00 \\ -3.196 & -0.928 & -1.100 & 1.00 & 0.00 \\ -2.884 & -1.644 & 0.488 & 1.00 & 0.00 \\ -3.772 & -0.116 & 0.360 & 1.00 & 0.00 \\ 1.924 & 0.916 & -0.288 & 1.00 & 0.00 \\ 1.768 & -0.192 & -0.756 & 1.00 & 0.00 \\ 0.428 & -0.884 & -0.804 & 1.00 & 0.00 \\ 2.860 & -0.824 & -1.256 & 1.00 & 0.00 \\ 2.760 & -2.124 & -1.820 & 1.00 & 0.00 \\ 0.472 & -1.840 & -0.268 & 1.00 & 0.00 \\ 0.168 & -1.136 & -1.840 & 1.00 & 0.00 \\ 2.416 & -2.860 & -1.084 & 1.00 & 0.00 \\ 2.100 & -2.136 & -2.696 & 1.00 & 0.00\end{array}$




\begin{tabular}{|c|c|c|c|c|c|c|c|c|c|}
\hline HETATM & 23 & $\mathrm{OH} 4$ & UNK & 0 & 3.776 & -2.384 & -2.136 & 1.00 & 0.00 \\
\hline TER & 24 & & UNK & 0 & & & & & \\
\hline \multicolumn{10}{|l|}{ END } \\
\hline \multicolumn{10}{|l|}{$7-B r$} \\
\hline HETATM & 1 & SN1 & UNK & 0 & 2.620 & 0.092 & -0.112 & 1.00 & 0.00 \\
\hline HETATM & 2 & BR2 & UNK & 0 & 5.464 & 0.156 & -0.040 & 1.00 & 0.00 \\
\hline HETATM & 3 & 03 & UNK & 0 & 0.476 & 0.184 & -0.180 & 1.00 & 0.00 \\
\hline HETATM & 4 & C4 & UNK & 0 & -0.436 & -0.744 & 0.008 & 1.00 & 0.00 \\
\hline HETATM & 5 & C5 & UNK & 0 & -1.844 & -0.220 & -0.044 & 1.00 & 0.00 \\
\hline HETATM & 6 & $\mathrm{C} 6$ & UNK & 0 & -2.964 & -1.040 & -0.252 & 1.00 & 0.00 \\
\hline HETATM & 7 & C7 & UNK & 0 & -4.252 & -0.504 & -0.276 & 1.00 & 0.00 \\
\hline HETATM & 8 & $\mathrm{C} 8$ & UNK & 0 & -4.456 & 0.864 & -0.100 & 1.00 & 0.00 \\
\hline HETATM & 9 & $\mathrm{C} 9$ & UNK & 0 & -3.348 & 1.692 & 0.092 & 1.00 & 0.00 \\
\hline HETATM & 10 & $\mathrm{C} 10$ & UNK & 0 & -2.060 & 1.156 & 0.112 & 1.00 & 0.00 \\
\hline HETATM & 11 & $1 \mathrm{H1}$ & UNK & 0 & -1.188 & 1.792 & 0.248 & 1.00 & 0.00 \\
\hline HETATM & 12 & $2 \mathrm{HI}$ & UNK & 0 & -3.484 & 2.764 & 0.220 & 1.00 & 0.00 \\
\hline HETATM & 13 & $3 \mathrm{H1}$ & UNK & 0 & -5.460 & 1.280 & -0.120 & 1.00 & 0.00 \\
\hline HETATM & 14 & $4 \mathrm{HI}$ & UNK & 0 & -5.104 & -1.164 & -0.448 & 1.00 & 0.00 \\
\hline HETATM & 15 & $5 \mathrm{HI}$ & UNK & 0 & -2.828 & -2.104 & -0.416 & 1.00 & 0.00 \\
\hline HETATM & 16 & C16 & UNK & 0 & -0.200 & -2.060 & 0.256 & 1.00 & 0.00 \\
\hline HETATM & 17 & 7H1 & UNK & 0 & -1.004 & -2.760 & 0.456 & 1.00 & 0.00 \\
\hline HETATM & 18 & $8 \mathrm{HI}$ & UNK & 0 & 0.812 & -2.452 & 0.300 & 1.00 & 0.00 \\
\hline HETATM & 19 & C19 & UNK & 0 & 2.636 & 2.188 & -0.572 & 1.00 & 0.00 \\
\hline HETATM & 20 & $\mathrm{OH} 2$ & UNK & 0 & 3.076 & 2.748 & 0.256 & 1.00 & 0.00 \\
\hline HETATM & 21 & $1 \mathrm{H} 2$ & UNK & 0 & 3.256 & 2.368 & -1.456 & 1.00 & 0.00 \\
\hline HETATM & 22 & $2 \mathrm{H} 2$ & UNK & 0 & 1.612 & 2.532 & -0.756 & 1.00 & 0.00 \\
\hline HETATM & 23 & C23 & UNK & 0 & 2.764 & -1.320 & -1.720 & 1.00 & 0.00 \\
\hline HETATM & 24 & $4 \mathrm{H} 2$ & UNK & 0 & 1.768 & -1.616 & -2.064 & 1.00 & 0.00 \\
\hline HETATM & 25 & $5 \mathrm{H} 2$ & UNK & 0 & 3.324 & -0.868 & -2.544 & 1.00 & 0.00 \\
\hline HETATM & 26 & $6 \mathrm{H} 2$ & UNK & 0 & 3.320 & -2.204 & -1.388 & 1.00 & 0.00 \\
\hline HETATM & 27 & C27 & UNK & 0 & 2.656 & -0.540 & 1.936 & 1.00 & 0.00 \\
\hline HETATM & 28 & $8 \mathrm{H} 2$ & UNK & 0 & 1.640 & -0.716 & 2.304 & 1.00 & 0.00 \\
\hline HETATM & 29 & $9 \mathrm{H} 2$ & UNK & 0 & 3.248 & -1.456 & 2.036 & 1.00 & 0.00 \\
\hline HETATM & 30 & $\mathrm{OH} 3$ & UNK & 0 & 3.144 & 0.232 & 2.540 & 1.00 & 0.00 \\
\hline TER & 31 & & UNK & 0 & & & & & \\
\hline \multicolumn{10}{|l|}{ END } \\
\hline \multicolumn{10}{|l|}{ A } \\
\hline ATOM & 1 & SN & UNK & 1 & 0.880 & 1.620 & 0.008 & 1.00 & 0.00 \\
\hline ATOM & 2 & 0 & UNK & 1 & -0.708 & 0.364 & -0.128 & 1.00 & 0.00 \\
\hline ATOM & 3 & C & UNK & 1 & -0.960 & -0.852 & 0.300 & 1.00 & 0.00 \\
\hline ATOM & 4 & C & UNK & 1 & -2.364 & -1.276 & 0.168 & 1.00 & 0.00 \\
\hline ATOM & 5 & C & UNK & 1 & -3.328 & -0.352 & -0.264 & 1.00 & 0.00 \\
\hline ATOM & 6 & C & UNK & 1 & -4.664 & -0.716 & -0.388 & 1.00 & 0.00 \\
\hline ATOM & 7 & C & UNK & 1 & -5.068 & -2.020 & -0.088 & 1.00 & 0.00 \\
\hline
\end{tabular}




\begin{tabular}{|c|c|c|c|c|c|c|c|c|c|}
\hline ATOM & 8 & $\mathrm{C}$ & UNK & 1 & -4.120 & -2.956 & 0.332 & 1.00 & 0.00 \\
\hline ATOM & 9 & $\mathrm{C}$ & UNK & 1 & -2.784 & -2.588 & 0.464 & 1.00 & 0.00 \\
\hline ATOM & 10 & $\mathrm{H}$ & UNK & 1 & -2.064 & -3.336 & 0.792 & 1.00 & 0.00 \\
\hline ATOM & 11 & $\mathrm{H}$ & UNK & 1 & -4.420 & -3.972 & 0.560 & 1.00 & 0.00 \\
\hline ATOM & 12 & $\mathrm{H}$ & UNK & 1 & -6.112 & -2.308 & -0.188 & 1.00 & 0.00 \\
\hline ATOM & 13 & $\mathrm{H}$ & UNK & 1 & -5.396 & 0.012 & -0.720 & 1.00 & 0.00 \\
\hline ATOM & 14 & $\mathrm{H}$ & UNK & 1 & -3.016 & 0.660 & -0.500 & 1.00 & 0.00 \\
\hline ATOM & 15 & $\mathrm{C}$ & UNK & 1 & 0.080 & -1.732 & 0.668 & 1.00 & 0.00 \\
\hline ATOM & 16 & $\mathrm{C}$ & UNK & 1 & 0.780 & -2.464 & -0.892 & 1.00 & 0.00 \\
\hline ATOM & 17 & $\mathrm{C}$ & UNK & 1 & 1.924 & -3.232 & -0.588 & 1.00 & 0.00 \\
\hline ATOM & 18 & $\mathrm{C}$ & UNK & 1 & 3.136 & -2.564 & -0.248 & 1.00 & 0.00 \\
\hline ATOM & 19 & 0 & UNK & 1 & 4.172 & -3.416 & 0.004 & 1.00 & 0.00 \\
\hline ATOM & 20 & $\mathrm{C}$ & UNK & 1 & 5.408 & -2.796 & 0.340 & 1.00 & 0.00 \\
\hline ATOM & 21 & $\mathrm{H}$ & UNK & 1 & 5.764 & -2.156 & -0.468 & 1.00 & 0.00 \\
\hline ATOM & 22 & $\mathrm{H}$ & UNK & 1 & 5.312 & -2.192 & 1.248 & 1.00 & 0.00 \\
\hline ATOM & 23 & $\mathrm{H}$ & UNK & 1 & 6.112 & -3.616 & 0.508 & 1.00 & 0.00 \\
\hline АTOM & 24 & 0 & UNK & 1 & 3.280 & -1.332 & -0.156 & 1.00 & 0.00 \\
\hline ATOM & 25 & $\mathrm{H}$ & UNK & 1 & 1.864 & -4.308 & -0.448 & 1.00 & 0.00 \\
\hline ATOM & 26 & $\mathrm{H}$ & UNK & 1 & 0.964 & -1.524 & -1.416 & 1.00 & 0.00 \\
\hline АTOM & 27 & $\mathrm{H}$ & UNK & 1 & -0.072 & -3.008 & -1.300 & 1.00 & 0.00 \\
\hline ATOM & 28 & $\mathrm{H}$ & UNK & 1 & -0.196 & -2.608 & 1.248 & 1.00 & 0.00 \\
\hline ATOM & 29 & $\mathrm{H}$ & UNK & 1 & 0.988 & -1.256 & 1.028 & 1.00 & 0.00 \\
\hline ATOM & 30 & $\mathrm{C}$ & UNK & 1 & 1.908 & 1.232 & 1.840 & 1.00 & 0.00 \\
\hline ATOM & 31 & $\mathrm{H}$ & UNK & 1 & 2.424 & 2.144 & 2.172 & 1.00 & 0.00 \\
\hline ATOM & 32 & $\mathrm{H}$ & UNK & 1 & 1.208 & 0.932 & 2.628 & 1.00 & 0.00 \\
\hline ATOM & 33 & $\mathrm{H}$ & UNK & 1 & 2.644 & 0.440 & 1.684 & 1.00 & 0.00 \\
\hline ATOM & 34 & $\mathrm{C}$ & UNK & 1 & -0.220 & 3.452 & 0.056 & 1.00 & 0.00 \\
\hline ATOM & 35 & $\mathrm{H}$ & UNK & 1 & 0.456 & 4.312 & 0.080 & 1.00 & 0.00 \\
\hline ATOM & 36 & $\mathrm{H}$ & UNK & 1 & -0.852 & 3.540 & -0.832 & 1.00 & 0.00 \\
\hline ATOM & 37 & $\mathrm{H}$ & UNK & 1 & -0.864 & 3.496 & 0.940 & 1.00 & 0.00 \\
\hline ATOM & 38 & C & UNK & 1 & 1.984 & 1.352 & -1.788 & 1.00 & 0.00 \\
\hline ATOM & 39 & $\mathrm{H}$ & UNK & 1 & 2.604 & 2.236 & -1.984 & 1.00 & 0.00 \\
\hline ATOM & 40 & $\mathrm{H}$ & UNK & 1 & 2.636 & 0.476 & -1.676 & 1.00 & 0.00 \\
\hline ATOM & 41 & $\mathrm{H}$ & UNK & 1 & 1.308 & 1.208 & -2.632 & 1.00 & 0.00 \\
\hline TER & 42 & & UNK & 1 & & & & & \\
\hline
\end{tabular}

B

\begin{tabular}{|c|c|c|c|c|c|c|c|c|c|}
\hline HETATM & 1 & SN1 & UNK & 0 & 3.384 & 1.992 & 0.744 & 1.00 & 0.00 \\
\hline HETATM & 2 & $\mathrm{O} 2$ & UNK & 0 & -3.036 & 0.644 & -1.488 & 1.00 & 0.00 \\
\hline HETATM & 3 & $\mathrm{C} 3$ & UNK & 0 & -3.012 & -0.084 & -0.504 & 1.00 & 0.00 \\
\hline HETATM & 4 & $\mathrm{C} 4$ & UNK & 0 & -3.824 & -1.348 & -0.500 & 1.00 & 0.00 \\
\hline HETATM & 5 & $\mathrm{C} 5$ & UNK & 0 & -4.528 & -1.688 & -1.664 & 1.00 & 0.00 \\
\hline HETATM & 6 & $\mathrm{C} 6$ & UNK & 0 & -5.304 & -2.840 & -1.716 & 1.00 & 0.00 \\
\hline HETATM & 7 & C7 & UNK & 0 & -5.392 & -3.672 & -0.596 & 1.00 & 0.00 \\
\hline HETATM & 8 & $\mathrm{C} 8$ & UNK & 0 & -4.704 & -3.344 & 0.568 & 1.00 & 0.00 \\
\hline
\end{tabular}




\begin{tabular}{|c|c|c|c|c|c|c|c|c|c|}
\hline HETATM & 9 & $\mathrm{C9}$ & UNK & 0 & -3.924 & -2.188 & 0.616 & 1.00 & 0.00 \\
\hline НЕТАТМ & 10 & $\mathrm{OH} 1$ & UNK & 0 & -3.396 & -1.948 & 1.536 & 1.00 & 0.00 \\
\hline HETATM & 11 & $1 \mathrm{H} 1$ & UNK & 0 & -4.772 & -3.988 & 1.440 & 1.00 & 0.00 \\
\hline HETATM & 12 & $2 \mathrm{H} 1$ & UNK & 0 & -6.000 & -4.572 & -0.636 & 1.00 & 0.00 \\
\hline HETATM & 13 & $3 \mathrm{H} 1$ & UNK & 0 & -5.840 & -3.092 & -2.628 & 1.00 & 0.00 \\
\hline HETATM & 14 & $4 \mathrm{H} 1$ & UNK & 0 & -4.448 & -1.024 & -2.524 & 1.00 & 0.00 \\
\hline HETATM & 15 & C15 & UNK & 0 & -2.136 & 0.252 & 0.680 & 1.00 & 0.00 \\
\hline HETATM & 16 & $\mathrm{C} 16$ & UNK & 0 & -0.812 & -0.560 & 0.712 & 1.00 & 0.00 \\
\hline HETATM & 17 & $\mathrm{C} 17$ & UNK & 0 & 0.072 & -0.316 & -0.468 & 1.00 & 0.00 \\
\hline НЕTATM & 18 & C18 & UNK & 0 & 1.236 & 0.356 & -0.372 & 1.00 & 0.00 \\
\hline HETATM & 19 & 019 & UNK & 0 & 2.072 & 0.636 & -1.428 & 1.00 & 0.00 \\
\hline HETATM & 20 & $\mathrm{C} 20$ & UNK & 0 & 1.652 & 0.300 & -2.736 & 1.00 & 0.00 \\
\hline HETATM & 21 & $1 \mathrm{H} 2$ & UNK & 0 & 1.552 & -0.788 & -2.844 & 1.00 & 0.00 \\
\hline HETATM & 22 & $2 \mathrm{H} 2$ & UNK & 0 & 0.696 & 0.780 & -2.976 & 1.00 & 0.00 \\
\hline HETATM & 23 & $3 \mathrm{H} 2$ & UNK & 0 & 2.432 & 0.660 & -3.408 & 1.00 & 0.00 \\
\hline НETATM & 24 & $\mathrm{O} 24$ & UNK & 0 & 1.752 & 0.832 & 0.752 & 1.00 & 0.00 \\
\hline HETATM & 25 & $5 \mathrm{H} 2$ & UNK & 0 & -0.256 & -0.664 & -1.436 & 1.00 & 0.00 \\
\hline HETATM & 26 & $6 \mathrm{H} 2$ & UNK & 0 & -1.052 & -1.632 & 0.792 & 1.00 & 0.00 \\
\hline HETATM & 27 & $7 \mathrm{H} 2$ & UNK & 0 & -0.280 & -0.288 & 1.628 & 1.00 & 0.00 \\
\hline HETATM & 28 & $8 \mathrm{H} 2$ & UNK & 0 & -2.684 & 0.088 & 1.616 & 1.00 & 0.00 \\
\hline HETATM & 29 & $9 \mathrm{H} 2$ & UNK & 0 & -1.900 & 1.316 & 0.608 & 1.00 & 0.00 \\
\hline HETATM & 30 & C30 & UNK & 0 & 3.476 & 2.408 & 2.840 & 1.00 & 0.00 \\
\hline HETATM & 31 & $1 \mathrm{H} 3$ & UNK & 0 & 4.352 & 3.016 & 3.084 & 1.00 & 0.00 \\
\hline HETATM & 32 & $2 \mathrm{H} 3$ & UNK & 0 & 2.580 & 2.944 & 3.164 & 1.00 & 0.00 \\
\hline HETATM & 33 & $3 \mathrm{H} 3$ & UNK & 0 & 3.536 & 1.472 & 3.408 & 1.00 & 0.00 \\
\hline HETATM & 34 & C34 & UNK & 0 & 2.988 & 3.752 & -0.404 & 1.00 & 0.00 \\
\hline HETATM & 35 & $5 \mathrm{H} 3$ & UNK & 0 & 3.640 & 4.576 & -0.088 & 1.00 & 0.00 \\
\hline HETATM & 36 & $6 \mathrm{H3}$ & UNK & 0 & 3.156 & 3.568 & -1.468 & 1.00 & 0.00 \\
\hline HETATM & 37 & 7H3 & UNK & 0 & 1.948 & 4.064 & -0.268 & 1.00 & 0.00 \\
\hline HETATM & 38 & C38 & UNK & 0 & 5.068 & 0.848 & 0.088 & 1.00 & 0.00 \\
\hline HETATM & 39 & $9 \mathrm{H} 3$ & UNK & 0 & 5.028 & -0.156 & 0.524 & 1.00 & 0.00 \\
\hline HETATM & 40 & $\mathrm{OH} 4$ & UNK & 0 & 5.064 & 0.748 & -1.000 & 1.00 & 0.00 \\
\hline HETATM & 41 & $1 \mathrm{H} 4$ & UNK & 0 & 6.004 & 1.320 & 0.400 & 1.00 & 0.00 \\
\hline TER & 42 & & UNK & 0 & & & & & \\
\hline
\end{tabular}

END

$\begin{array}{lrrrr}\text { C } & & & & \\ \text { HETATM } & 1 & \text { SN1 } & \text { UNK } & 0 \\ \text { HETATM } & 2 & \text { BR2 } & \text { UNK } & 0 \\ \text { HETATM } & 3 & \text { O3 } & \text { UNK } & 0 \\ \text { HETATM } & 4 & \text { C4 } & \text { UNK } & 0 \\ \text { HETATM } & 5 & \text { C5 } & \text { UNK } & 0 \\ \text { HETATM } & 6 & \text { C6 } & \text { UNK } & 0 \\ \text { HETATM } & 7 & \text { C7 } & \text { UNK } & 0 \\ \text { HETATM } & 8 & \text { C8 } & \text { UNK } & 0 \\ \text { HETATM } & 9 & \text { C9 } & \text { UNK } & 0\end{array}$

$\begin{array}{rrrrr}1.716 & -0.604 & -0.180 & 1.00 & 0.00 \\ -1.072 & -1.304 & -0.360 & 1.00 & 0.00 \\ 3.752 & 0.032 & -0.108 & 1.00 & 0.00 \\ 4.832 & -0.588 & 0.320 & 1.00 & 0.00 \\ 6.088 & 0.216 & 0.168 & 1.00 & 0.00 \\ 6.088 & 1.312 & -0.704 & 1.00 & 0.00 \\ 7.236 & 2.084 & -0.892 & 1.00 & 0.00 \\ 8.408 & 1.784 & -0.200 & 1.00 & 0.00 \\ 8.416 & 0.704 & 0.684 & 1.00 & 0.00\end{array}$




\begin{tabular}{|c|c|c|c|c|c|c|c|c|c|}
\hline HETATM & 10 & $\mathrm{C} 10$ & UNK & 0 & 7.272 & -0.064 & 0.868 & 1.00 & 0.00 \\
\hline НЕТАТМ & 11 & $1 \mathrm{H} 1$ & UNK & 0 & 7.292 & -0.880 & 1.584 & 1.00 & 0.00 \\
\hline НЕТАТМ & 12 & $2 \mathrm{H} 1$ & UNK & 0 & 9.320 & 0.468 & 1.248 & 1.00 & 0.00 \\
\hline НЕТАТМ & 13 & $3 \mathrm{H} 1$ & UNK & 0 & 9.304 & 2.388 & -0.340 & 1.00 & 0.00 \\
\hline НЕТАТМ & 14 & $4 \mathrm{H} 1$ & UNK & 0 & 7.208 & 2.928 & -1.580 & 1.00 & 0.00 \\
\hline HETATM & 15 & $5 \mathrm{HI}$ & UNK & 0 & 5.164 & 1.544 & -1.224 & 1.00 & 0.00 \\
\hline НЕТАТМ & 16 & C16 & UNK & 0 & 4.872 & -1.848 & 0.836 & 1.00 & 0.00 \\
\hline НЕТАТМ & 17 & 7H1 & UNK & 0 & 5.804 & -2.316 & 1.124 & 1.00 & 0.00 \\
\hline НЕТАТМ & 18 & $8 \mathrm{H1}$ & UNK & 0 & 3.968 & -2.440 & 0.948 & 1.00 & 0.00 \\
\hline НЕTATM & 19 & C19 & UNK & 0 & 2.104 & -2.260 & -1.492 & 1.00 & 0.00 \\
\hline HETATM & 20 & OH2 & UNK & 0 & 1.552 & -2.112 & -2.428 & 1.00 & 0.00 \\
\hline НЕТАТМ & 21 & $1 \mathrm{H} 2$ & UNK & 0 & 3.176 & -2.340 & -1.700 & 1.00 & 0.00 \\
\hline HETATM & 22 & $2 \mathrm{H} 2$ & UNK & 0 & 1.744 & -3.188 & -1.036 & 1.00 & 0.00 \\
\hline HETATM & 23 & C23 & UNK & 0 & 1.204 & 1.304 & -1.012 & 1.00 & 0.00 \\
\hline HETATM & 24 & $4 \mathrm{H} 2$ & UNK & 0 & 0.452 & 1.788 & -0.388 & 1.00 & 0.00 \\
\hline HETATM & 25 & $5 \mathrm{H} 2$ & UNK & 0 & 2.100 & 1.928 & -1.068 & 1.00 & 0.00 \\
\hline HETATM & 26 & $6 \mathrm{H} 2$ & UNK & 0 & 0.776 & 1.172 & -2.012 & 1.00 & 0.00 \\
\hline НЕTATM & 27 & $\mathrm{C} 27$ & UNK & 0 & 1.548 & -0.896 & 1.940 & 1.00 & 0.00 \\
\hline НЕTATM & 28 & $8 \mathrm{H} 2$ & UNK & 0 & 0.776 & -0.228 & 2.332 & 1.00 & 0.00 \\
\hline НЕTATM & 29 & $9 \mathrm{H} 2$ & UNK & 0 & 1.236 & -1.924 & 2.148 & 1.00 & 0.00 \\
\hline НЕTATM & 30 & ОН3 & UNK & 0 & 2.504 & -0.692 & 2.428 & 1.00 & 0.00 \\
\hline HETATM & 31 & 031 & UNK & 0 & -7.532 & -0.404 & -0.144 & 1.00 & 0.00 \\
\hline HETATM & 32 & C32 & UNK & 0 & -6.804 & 0.540 & 0.100 & 1.00 & 0.00 \\
\hline HETATM & 33 & 033 & UNK & 0 & -5.468 & 0.492 & 0.088 & 1.00 & 0.00 \\
\hline НЕТАТМ & 34 & C34 & UNK & 0 & -4.868 & -0.772 & -0.240 & 1.00 & 0.00 \\
\hline НЕТАТМ & 35 & $5 \mathrm{H} 3$ & UNK & 0 & -5.180 & -1.528 & 0.484 & 1.00 & 0.00 \\
\hline HETATM & 36 & $6 \mathrm{H} 3$ & UNK & 0 & -5.180 & -1.080 & -1.240 & 1.00 & 0.00 \\
\hline HETATM & 37 & $7 \mathrm{H} 3$ & UNK & 0 & -3.788 & -0.620 & -0.200 & 1.00 & 0.00 \\
\hline HETATM & 38 & C38 & UNK & 0 & -7.268 & 1.904 & 0.460 & 1.00 & 0.00 \\
\hline HETATM & 39 & C39 & UNK & 0 & -8.572 & 2.196 & 0.540 & 1.00 & 0.00 \\
\hline HETATM & 40 & $\mathrm{OH} 4$ & UNK & 0 & -9.316 & 1.428 & 0.344 & 1.00 & 0.00 \\
\hline HETATM & 41 & $1 \mathrm{H} 4$ & UNK & 0 & -8.916 & 3.192 & 0.804 & 1.00 & 0.00 \\
\hline HETATM & 42 & $2 \mathrm{H} 4$ & UNK & 0 & -6.500 & 2.648 & 0.652 & 1.00 & 0.00 \\
\hline TER & 43 & & UNK & 0 & & & & & \\
\hline
\end{tabular}

END

D

$\begin{array}{lrrrrrrrrr}\text { HETATM } & 1 & \text { SN1 } & \text { UNK } & 0 & 2.276 & 0.116 & -0.228 & 1.00 & 0.00 \\ \text { HETATM } & 2 & \text { BR2 } & \text { UNK } & 0 & 4.772 & -0.976 & 0.128 & 1.00 & 0.00 \\ \text { HETATM } & 3 & \text { O3 } & \text { UNK } & 0 & 0.296 & 1.204 & -0.496 & 1.00 & 0.00 \\ \text { HETATM } & 4 & \text { C4 } & \text { UNK } & 0 & -0.868 & 1.136 & 0.044 & 1.00 & 0.00 \\ \text { HETATM } & 5 & \text { C5 } & \text { UNK } & 0 & -1.656 & 2.408 & 0.032 & 1.00 & 0.00 \\ \text { HETATM } & 6 & \text { C6 } & \text { UNK } & 0 & -1.000 & 3.604 & -0.304 & 1.00 & 0.00 \\ \text { HETATM } & 7 & \text { C7 } & \text { UNK } & 0 & -1.680 & 4.820 & -0.316 & 1.00 & 0.00 \\ \text { HETATM } & 8 & \text { C8 } & \text { UNK } & 0 & -3.040 & 4.868 & -0.004 & 1.00 & 0.00 \\ \text { HETATM } & 9 & \text { C9 } & \text { UNK } & 0 & -3.708 & 3.688 & 0.320 & 1.00 & 0.00\end{array}$




\begin{tabular}{|c|c|c|c|c|c|c|c|c|c|}
\hline HETATM & 10 & $\mathrm{C} 10$ & UNK & 0 & -3.024 & 2.472 & 0.340 & 1.00 & 0.00 \\
\hline НЕTATM & 11 & $1 \mathrm{H1}$ & UNK & 0 & -3.568 & 1.564 & 0.584 & 1.00 & 0.00 \\
\hline HETATM & 12 & $2 \mathrm{H} 1$ & UNK & 0 & -4.772 & 3.708 & 0.556 & 1.00 & 0.00 \\
\hline HETATM & 13 & $3 \mathrm{H1}$ & UNK & 0 & -3.572 & 5.816 & -0.016 & 1.00 & 0.00 \\
\hline HETATM & 14 & $4 \mathrm{H} 1$ & UNK & 0 & -1.148 & 5.732 & -0.576 & 1.00 & 0.00 \\
\hline НЕТАТМ & 15 & $5 \mathrm{HI}$ & UNK & 0 & 0.056 & 3.552 & -0.556 & 1.00 & 0.00 \\
\hline HETATM & 16 & C16 & UNK & 0 & -1.456 & -0.048 & 0.516 & 1.00 & 0.00 \\
\hline HETATM & 17 & $\mathrm{C} 17$ & UNK & 0 & -2.412 & -0.884 & -1.092 & 1.00 & 0.00 \\
\hline HETATM & 18 & $\mathrm{C} 18$ & UNK & 0 & -3.352 & -1.832 & -0.688 & 1.00 & 0.00 \\
\hline HETATM & 19 & C19 & UNK & 0 & -2.960 & -3.168 & -0.356 & 1.00 & 0.00 \\
\hline HETATM & 20 & 020 & UNK & 0 & -4.040 & -3.944 & 0.028 & 1.00 & 0.00 \\
\hline HETATM & 21 & $\mathrm{C} 21$ & UNK & 0 & -3.720 & -5.288 & 0.348 & 1.00 & 0.00 \\
\hline HETATM & 22 & $2 \mathrm{H} 2$ & UNK & 0 & -3.292 & -5.812 & -0.508 & 1.00 & 0.00 \\
\hline HETATM & 23 & $3 \mathrm{H} 2$ & UNK & 0 & -3.004 & -5.340 & 1.176 & 1.00 & 0.00 \\
\hline НЕТАТМ & 24 & $4 \mathrm{H} 2$ & UNK & 0 & -4.668 & -5.756 & 0.640 & 1.00 & 0.00 \\
\hline НЕТАТМ & 25 & 025 & UNK & 0 & -1.828 & -3.648 & -0.384 & 1.00 & 0.00 \\
\hline HETATM & 26 & $6 \mathrm{H} 2$ & UNK & 0 & -4.388 & -1.556 & -0.512 & 1.00 & 0.00 \\
\hline HETATM & 27 & $7 \mathrm{H} 2$ & UNK & 0 & -1.480 & -1.236 & -1.524 & 1.00 & 0.00 \\
\hline HETATM & 28 & $8 \mathrm{H} 2$ & UNK & 0 & -2.788 & 0.036 & -1.532 & 1.00 & 0.00 \\
\hline HETATM & 29 & $9 \mathrm{H} 2$ & UNK & 0 & -2.304 & 0.024 & 1.184 & 1.00 & 0.00 \\
\hline HETATM & 30 & OH3 & UNK & 0 & -0.800 & -0.892 & 0.708 & 1.00 & 0.00 \\
\hline HETATM & 31 & C31 & UNK & 0 & 1.960 & 0.080 & 1.892 & 1.00 & 0.00 \\
\hline HETATM & 32 & $2 \mathrm{H} 3$ & UNK & 0 & 2.772 & 0.632 & 2.372 & 1.00 & 0.00 \\
\hline HETATM & 33 & $3 \mathrm{H} 3$ & UNK & 0 & 0.996 & 0.524 & 2.164 & 1.00 & 0.00 \\
\hline HETATM & 34 & $4 \mathrm{H} 3$ & UNK & 0 & 2.000 & -0.948 & 2.248 & 1.00 & 0.00 \\
\hline HETATM & 35 & C35 & UNK & 0 & 3.124 & 1.856 & -1.152 & 1.00 & 0.00 \\
\hline HETATM & 36 & $6 \mathrm{H3}$ & UNK & 0 & 3.884 & 1.552 & -1.880 & 1.00 & 0.00 \\
\hline HETATM & 37 & 7H3 & UNK & 0 & 2.340 & 2.436 & -1.648 & 1.00 & 0.00 \\
\hline HETATM & 38 & $8 \mathrm{H} 3$ & UNK & 0 & 3.616 & 2.480 & -0.396 & 1.00 & 0.00 \\
\hline HETATM & 39 & C39 & UNK & 0 & 1.600 & -1.452 & -1.520 & 1.00 & 0.00 \\
\hline HETATM & 40 & $\mathrm{OH} 4$ & UNK & 0 & 2.460 & -2.028 & -1.872 & 1.00 & 0.00 \\
\hline HETATM & 41 & $1 \mathrm{H} 4$ & UNK & 0 & 0.912 & -2.124 & -1.000 & 1.00 & 0.00 \\
\hline HETATM & 42 & $2 \mathrm{H} 4$ & UNK & 0 & 1.076 & -1.008 & -2.372 & 1.00 & 0.00 \\
\hline $\mathrm{TER}$ & 43 & & UNK & 0 & & & & & \\
\hline
\end{tabular}

END

$\mathbf{E}$
HETATM
HETATM
HETATM
HETATM
HETATM
HETATM
HETATM
HETATM
HETATM

$\begin{array}{rrrl}1 & \text { SN1 } & \text { UNK } & 0 \\ 2 & \text { BR2 } & \text { UNK } & 0 \\ 3 & \text { O3 } & \text { UNK } & 0 \\ 4 & \text { C4 } & \text { UNK } & 0 \\ 5 & \text { C5 } & \text { UNK } & 0 \\ 6 & \text { C6 } & \text { UNK } & 0 \\ 7 & \text { C7 } & \text { UNK } & 0 \\ 8 & \text { C8 } & \text { UNK } & 0 \\ 9 & \text { C9 } & \text { UNK } & 0\end{array}$

$$
\begin{array}{r}
2.284 \\
4.664 \\
0.220 \\
-0.952 \\
-1.636 \\
-0.884 \\
-1.472 \\
-2.836 \\
-3.600
\end{array}
$$

$\begin{array}{rrrr}-0.208 & -0.204 & 1.00 & 0.00 \\ -1.392 & 0.112 & 1.00 & 0.00 \\ 1.096 & -0.448 & 1.00 & 0.00 \\ 1.136 & -0.000 & 1.00 & 0.00 \\ 2.456 & 0.016 & 1.00 & 0.00 \\ 3.612 & -0.256 & 1.00 & 0.00 \\ 4.872 & -0.236 & 1.00 & 0.00 \\ 5.008 & 0.044 & 1.00 & 0.00 \\ 3.868 & 0.308 & 1.00 & 0.00\end{array}$




\begin{tabular}{|c|c|c|c|c|c|c|c|c|c|}
\hline HETATM & 10 & $\mathrm{C} 10$ & UNK & 0 & -3.008 & 2.604 & 0.296 & 1.00 & 0.00 \\
\hline НЕТАТМ & 11 & $1 \mathrm{H} 1$ & UNK & 0 & -3.620 & 1.732 & 0.496 & 1.00 & 0.00 \\
\hline НЕТАТМ & 12 & $2 \mathrm{H} 1$ & UNK & 0 & -4.664 & 3.960 & 0.520 & 1.00 & 0.00 \\
\hline НЕТАТМ & 13 & $3 \mathrm{H} 1$ & UNK & 0 & -3.300 & 5.992 & 0.056 & 1.00 & 0.00 \\
\hline НЕТАТМ & 14 & $4 \mathrm{H} 1$ & UNK & 0 & -0.872 & 5.752 & -0.448 & 1.00 & 0.00 \\
\hline НЕТАТМ & 15 & $5 \mathrm{H} 1$ & UNK & 0 & 0.172 & 3.492 & -0.480 & 1.00 & 0.00 \\
\hline HETATM & 16 & C16 & UNK & 0 & -1.692 & -0.084 & 0.360 & 1.00 & 0.00 \\
\hline НЕТАТМ & 17 & $\mathrm{C} 17$ & UNK & 0 & -2.392 & -0.728 & -0.952 & 1.00 & 0.00 \\
\hline HETATM & 18 & $\mathrm{C} 18$ & UNK & 0 & -3.224 & -1.896 & -0.624 & 1.00 & 0.00 \\
\hline НЕTATM & 19 & C19 & UNK & 0 & -2.612 & -3.084 & -0.216 & 1.00 & 0.00 \\
\hline HETATM & 20 & 020 & UNK & 0 & -3.532 & -4.092 & 0.116 & 1.00 & 0.00 \\
\hline HETATM & 21 & $\mathrm{C} 21$ & UNK & 0 & -2.944 & -5.316 & 0.512 & 1.00 & 0.00 \\
\hline HETATM & 22 & $2 \mathrm{H} 2$ & UNK & 0 & -2.328 & -5.748 & -0.284 & 1.00 & 0.00 \\
\hline НЕТАТМ & 23 & $3 \mathrm{H} 2$ & UNK & 0 & -2.312 & -5.196 & 1.400 & 1.00 & 0.00 \\
\hline HETATM & 24 & $4 \mathrm{H} 2$ & UNK & 0 & -3.780 & -5.988 & 0.740 & 1.00 & 0.00 \\
\hline HETATM & 25 & 025 & UNK & 0 & -1.392 & -3.324 & -0.128 & 1.00 & 0.00 \\
\hline HETATM & 26 & $6 \mathrm{H} 2$ & UNK & 0 & -4.304 & -1.796 & -0.540 & 1.00 & 0.00 \\
\hline HETATM & 27 & $7 \mathrm{H} 2$ & UNK & 0 & -1.548 & -0.980 & -1.604 & 1.00 & 0.00 \\
\hline HETATM & 28 & $8 \mathrm{H} 2$ & UNK & 0 & -2.984 & 0.056 & -1.444 & 1.00 & 0.00 \\
\hline HETATM & 29 & $9 \mathrm{H} 2$ & UNK & 0 & -2.476 & 0.096 & 1.100 & 1.00 & 0.00 \\
\hline HETATM & 30 & ОН3 & UNK & 0 & -1.020 & -0.864 & 0.728 & 1.00 & 0.00 \\
\hline HETATM & 31 & C31 & UNK & 0 & 1.808 & -0.300 & 1.884 & 1.00 & 0.00 \\
\hline HETATM & 32 & $2 \mathrm{H} 3$ & UNK & 0 & 2.700 & -0.044 & 2.460 & 1.00 & 0.00 \\
\hline HETATM & 33 & $3 \mathrm{H3}$ & UNK & 0 & 0.992 & 0.380 & 2.140 & 1.00 & 0.00 \\
\hline HETATM & 34 & $4 \mathrm{H} 3$ & UNK & 0 & 1.516 & -1.324 & 2.140 & 1.00 & 0.00 \\
\hline HETATM & 35 & C35 & UNK & 0 & 3.124 & 1.612 & -0.968 & 1.00 & 0.00 \\
\hline HЕTATM & 36 & $6 \mathrm{H3}$ & UNK & 0 & 3.992 & 1.380 & -1.592 & 1.00 & 0.00 \\
\hline HЕTATM & 37 & $7 \mathrm{H3}$ & UNK & 0 & 2.372 & 2.152 & -1.552 & 1.00 & 0.00 \\
\hline HETATM & 38 & $8 \mathrm{H3}$ & UNK & 0 & 3.456 & 2.248 & -0.136 & 1.00 & 0.00 \\
\hline HETATM & 39 & C39 & UNK & 0 & 1.460 & -1.596 & -1.600 & 1.00 & 0.00 \\
\hline HETATM & 40 & $\mathrm{OH} 4$ & UNK & 0 & 2.240 & -2.292 & -1.924 & 1.00 & 0.00 \\
\hline HETATM & 41 & $1 \mathrm{H} 4$ & UNK & 0 & 0.640 & -2.160 & -1.140 & 1.00 & 0.00 \\
\hline HETATM & 42 & $2 \mathrm{H} 4$ & UNK & 0 & 1.072 & -1.052 & -2.464 & 1.00 & 0.00 \\
\hline $\mathrm{TER}$ & 43 & & UNK & 0 & & & & & \\
\hline
\end{tabular}

END

$\begin{array}{lrrrr}\text { F } & & & & \\ \text { ATOM } & 1 & \text { SN } & \text { UNK } & 1 \\ \text { ATOM } & 2 & \text { BR } & \text { UNK } & 1 \\ \text { ATOM } & 3 & \text { O } & \text { UNK } & 1 \\ \text { ATOM } & 4 & \text { C } & \text { UNK } & 1 \\ \text { ATOM } & 5 & \text { C } & \text { UNK } & 1 \\ \text { ATOM } & 6 & \text { C } & \text { UNK } & 1 \\ \text { ATOM } & 7 & \text { C } & \text { UNK } & 1 \\ \text { ATOM } & 8 & \text { C } & \text { UNK } & 1 \\ \text { ATOM } & 9 & \text { C } & \text { UNK } & 1\end{array}$

$\begin{array}{rrrrr}-3.572 & -1.532 & -0.092 & 1.00 & 0.00 \\ -6.032 & -2.304 & -0.072 & 1.00 & 0.00 \\ 0.288 & -2.356 & 0.100 & 1.00 & 0.00 \\ 1.356 & -1.744 & 0.244 & 1.00 & 0.00 \\ 2.644 & -2.508 & 0.112 & 1.00 & 0.00 \\ 2.588 & -3.908 & 0.048 & 1.00 & 0.00 \\ 3.748 & -4.668 & -0.064 & 1.00 & 0.00 \\ 4.996 & -4.040 & -0.124 & 1.00 & 0.00 \\ 5.068 & -2.648 & -0.072 & 1.00 & 0.00\end{array}$




\begin{tabular}{|c|c|c|c|c|c|c|c|c|c|}
\hline ATOM & 10 & $\mathrm{C}$ & UNK & 1 & 3.904 & -1.888 & 0.048 & 1.00 & 0.00 \\
\hline ATOM & 11 & $\mathrm{H}$ & UNK & 1 & 3.976 & -0.808 & 0.080 & 1.00 & 0.00 \\
\hline ATOM & 12 & $\mathrm{H}$ & UNK & 1 & 6.032 & -2.148 & -0.128 & 1.00 & 0.00 \\
\hline ATOM & 13 & $\mathrm{H}$ & UNK & 1 & 5.904 & -4.632 & -0.216 & 1.00 & 0.00 \\
\hline ATOM & 14 & $\mathrm{H}$ & UNK & 1 & 3.684 & -5.756 & -0.104 & 1.00 & 0.00 \\
\hline ATOM & 15 & $\mathrm{H}$ & UNK & 1 & 1.608 & -4.380 & 0.084 & 1.00 & 0.00 \\
\hline ATOM & 16 & $\mathrm{C}$ & UNK & 1 & 1.380 & -0.272 & 0.432 & 1.00 & 0.00 \\
\hline ATOM & 17 & $\mathrm{C}$ & UNK & 1 & 1.236 & 0.488 & -0.960 & 1.00 & 0.00 \\
\hline ATOM & 18 & $\mathrm{C}$ & UNK & 1 & 1.256 & 1.960 & -0.820 & 1.00 & 0.00 \\
\hline ATOM & 19 & $\mathrm{C}$ & UNK & 1 & 0.180 & 2.616 & -0.236 & 1.00 & 0.00 \\
\hline ATOM & 20 & 0 & UNK & 1 & 0.360 & 4.008 & -0.116 & 1.00 & 0.00 \\
\hline ATOM & 21 & $\mathrm{C}$ & UNK & 1 & -0.732 & 4.704 & 0.432 & 1.00 & 0.00 \\
\hline ATOM & 22 & $\mathrm{H}$ & UNK & 1 & -1.636 & 4.600 & -0.180 & 1.00 & 0.00 \\
\hline ATOM & 23 & $\mathrm{H}$ & UNK & 1 & -0.972 & 4.356 & 1.444 & 1.00 & 0.00 \\
\hline ATOM & 24 & $\mathrm{H}$ & UNK & 1 & -0.432 & 5.756 & 0.464 & 1.00 & 0.00 \\
\hline ATOM & 25 & 0 & UNK & 1 & -0.896 & 2.120 & 0.184 & 1.00 & 0.00 \\
\hline АTOM & 26 & $\mathrm{H}$ & UNK & 1 & 2.156 & 2.524 & -1.064 & 1.00 & 0.00 \\
\hline ATOM & 27 & $\mathrm{H}$ & UNK & 1 & 0.292 & 0.120 & -1.384 & 1.00 & 0.00 \\
\hline ATOM & 28 & $\mathrm{H}$ & UNK & 1 & 2.044 & 0.148 & -1.624 & 1.00 & 0.00 \\
\hline АTOM & 29 & $\mathrm{H}$ & UNK & 1 & 2.288 & 0.080 & 0.928 & 1.00 & 0.00 \\
\hline ATOM & 30 & $\mathrm{H}$ & UNK & 1 & 0.508 & 0.036 & 1.020 & 1.00 & 0.00 \\
\hline ATOM & 31 & $\mathrm{C}$ & UNK & 1 & -2.904 & -2.036 & 1.872 & 1.00 & 0.00 \\
\hline ATOM & 32 & $\mathrm{H}$ & UNK & 1 & -3.216 & -3.052 & 2.128 & 1.00 & 0.00 \\
\hline ATOM & 33 & $\mathrm{H}$ & UNK & 1 & -1.812 & -1.976 & 1.892 & 1.00 & 0.00 \\
\hline ATOM & 34 & $\mathrm{H}$ & UNK & 1 & -3.324 & -1.340 & 2.608 & 1.00 & 0.00 \\
\hline ATOM & 35 & $\mathrm{C}$ & UNK & 1 & -2.724 & -2.760 & -1.628 & 1.00 & 0.00 \\
\hline ATOM & 36 & $\mathrm{H}$ & UNK & 1 & -3.072 & -2.436 & -2.612 & 1.00 & 0.00 \\
\hline ATOM & 37 & $\mathrm{H}$ & UNK & 1 & -1.632 & -2.684 & -1.572 & 1.00 & 0.00 \\
\hline ATOM & 38 & $\mathrm{H}$ & UNK & 1 & -3.020 & -3.804 & -1.476 & 1.00 & 0.00 \\
\hline ATOM & 39 & $\mathrm{C}$ & UNK & 1 & -3.700 & 0.544 & -0.528 & 1.00 & 0.00 \\
\hline АTOM & 40 & $\mathrm{H}$ & UNK & 1 & -4.436 & 1.008 & 0.136 & 1.00 & 0.00 \\
\hline ATOM & 41 & $\mathrm{H}$ & UNK & 1 & -2.724 & 1.032 & -0.380 & 1.00 & 0.00 \\
\hline ATOM & 42 & $\mathrm{H}$ & UNK & 1 & -4.028 & 0.688 & -1.560 & 1.00 & 0.00 \\
\hline TER & 43 & & UNK & 1 & & & & & \\
\hline
\end{tabular}

G

$\begin{array}{lllllrrrrr}\text { HETATM } & 1 & \mathrm{O} & 2 & 2 & -2.988 & 0.852 & -1.612 & 1.00 & 0.00 \\ \text { HETATM } & 2 & \mathrm{C} & 2 & 2 & -2.428 & 0.256 & -0.704 & 1.00 & 0.00 \\ \text { HETATM } & 3 & \mathrm{C} & 2 & 2 & -3.044 & -1.008 & -0.168 & 1.00 & 0.00 \\ \text { HETATM } & 4 & \mathrm{C} & 2 & 2 & -2.428 & -1.792 & 0.816 & 1.00 & 0.00 \\ \text { HETATM } & 5 & \mathrm{C} & 2 & 2 & -3.036 & -2.956 & 1.280 & 1.00 & 0.00 \\ \text { HETATM } & 6 & \mathrm{C} & 2 & 2 & -4.272 & -3.356 & 0.764 & 1.00 & 0.00 \\ \text { HETATM } & 7 & \mathrm{C} & 2 & 2 & -4.892 & -2.580 & -0.220 & 1.00 & 0.00 \\ \text { HETATM } & 8 & \mathrm{C} & 2 & 2 & -4.280 & -1.420 & -0.680 & 1.00 & 0.00 \\ \text { HETATM } & 9 & \mathrm{H} & 2 & 2 & -4.744 & -0.804 & -1.448 & 1.00 & 0.00\end{array}$




\begin{tabular}{|c|c|c|c|c|c|c|c|c|c|}
\hline HETATM & 10 & $\mathrm{H}$ & 2 & 2 & -5.856 & -2.888 & -0.624 & 1.00 & 0.00 \\
\hline HETATM & 11 & $\mathrm{H}$ & 2 & 2 & -4.744 & -4.264 & 1.124 & 1.00 & 0.00 \\
\hline HETATM & 12 & $\mathrm{H}$ & 2 & 2 & -2.544 & -3.556 & 2.040 & 1.00 & 0.00 \\
\hline HETATM & 13 & $\mathrm{H}$ & 2 & 2 & -1.464 & -1.496 & 1.224 & 1.00 & 0.00 \\
\hline HETATM & 14 & C & 2 & 2 & -1.144 & 0.752 & -0.084 & 1.00 & 0.00 \\
\hline HETATM & 15 & C & 2 & 2 & -0.544 & 2.008 & -0.724 & 1.00 & 0.00 \\
\hline HETATM & 16 & C & 2 & 2 & 0.668 & 2.516 & 0.004 & 1.00 & 0.00 \\
\hline HETATM & 17 & C & 2 & 2 & 1.808 & 1.772 & 0.080 & 1.00 & 0.00 \\
\hline HETATM & 18 & 0 & 2 & 2 & 1.964 & 0.596 & -0.440 & 1.00 & 0.00 \\
\hline HETATM & 19 & SN & 2 & 2 & 3.696 & -0.716 & -0.268 & 1.00 & 0.00 \\
\hline HETATM & 20 & $\mathrm{BR}$ & 2 & 2 & 5.856 & -2.548 & -0.100 & 1.00 & 0.00 \\
\hline HETATM & 21 & C & 2 & 2 & 2.612 & -2.136 & -1.460 & 1.00 & 0.00 \\
\hline HETATM & 22 & $\mathrm{H}$ & 2 & 2 & 3.216 & -2.420 & -2.328 & 1.00 & 0.00 \\
\hline HETATM & 23 & $\mathrm{H}$ & 2 & 2 & 2.424 & -3.044 & -0.876 & 1.00 & 0.00 \\
\hline HETATM & 24 & $\mathrm{H}$ & 2 & 2 & 1.668 & -1.700 & -1.788 & 1.00 & 0.00 \\
\hline HETATM & 25 & C & 2 & 2 & 3.536 & -0.840 & 1.868 & 1.00 & 0.00 \\
\hline HETATM & 26 & $\mathrm{H}$ & 2 & 2 & 3.528 & -1.896 & 2.164 & 1.00 & 0.00 \\
\hline HETATM & 27 & $\mathrm{H}$ & 2 & 2 & 4.408 & -0.372 & 2.328 & 1.00 & 0.00 \\
\hline HETATM & 28 & $\mathrm{H}$ & 2 & 2 & 2.628 & -0.344 & 2.212 & 1.00 & 0.00 \\
\hline HETATM & 29 & $\mathrm{C}$ & 2 & 2 & 5.012 & 0.640 & -1.284 & 1.00 & 0.00 \\
\hline HETATM & 30 & $\mathrm{H}$ & 2 & 2 & 5.784 & 0.992 & -0.588 & 1.00 & 0.00 \\
\hline HETATM & 31 & $\mathrm{H}$ & 2 & 2 & 5.512 & 0.112 & -2.104 & 1.00 & 0.00 \\
\hline HETATM & 32 & $\mathrm{H}$ & 2 & 2 & 4.448 & 1.496 & -1.664 & 1.00 & 0.00 \\
\hline HETATM & 33 & 0 & 2 & 2 & 2.948 & 2.204 & 0.740 & 1.00 & 0.00 \\
\hline HETATM & 34 & C & 2 & 2 & 2.932 & 3.464 & 1.360 & 1.00 & 0.00 \\
\hline HETATM & 35 & $\mathrm{H}$ & 2 & 2 & 2.164 & 3.516 & 2.148 & 1.00 & 0.00 \\
\hline HETATM & 36 & $\mathrm{H}$ & 2 & 2 & 3.920 & 3.596 & 1.808 & 1.00 & 0.00 \\
\hline HETATM & 37 & $\mathrm{H}$ & 2 & 2 & 2.748 & 4.268 & 0.636 & 1.00 & 0.00 \\
\hline HETATM & 38 & $\mathrm{H}$ & 2 & 2 & 0.616 & 3.492 & 0.468 & 1.00 & 0.00 \\
\hline HETATM & 39 & $\mathrm{H}$ & 2 & 2 & -0.308 & 1.768 & -1.772 & 1.00 & 0.00 \\
\hline HETATM & 40 & $\mathrm{H}$ & 2 & 2 & -1.312 & 2.792 & -0.764 & 1.00 & 0.00 \\
\hline HETATM & 41 & $\mathrm{H}$ & 2 & 2 & -0.388 & -0.040 & -0.108 & 1.00 & 0.00 \\
\hline HETATM & 42 & $\mathrm{H}$ & 2 & 2 & -1.336 & 0.936 & 0.984 & 1.00 & 0.00 \\
\hline TER & 43 & & 2 & 2 & & & & & \\
\hline
\end{tabular}

END 